\title{
L'EMPRESA DE LA CORREGE ET LA CONQUETE DE LA SICILE: LE ROYAUME ERRANT DE MARTIN DE MONTBLANC
}

\author{
HENRI BRESC \\ Université de Paris-Nanterre
}

\begin{abstract}
SUMARI
1. Les statuts de l'Empresa: texte et contexte. - 2. Le jeu, l'honneur, la gratuité.

- 3. De la principauté en marche au hochet mondain. - 4. Texte des Statuts.
\end{abstract}

Expansion de la Couronne d'Aragon et conquête de la Sicile ne sont pas seulement, à fin du XIve siècle, la réalisation d'un programme rationnel, fondé sur les revendications dynastiques et appuyé sur la conscience lucide des chances qu'offre à la Catalogne la mise en ouvre d'une complémentarité entre blé sicilien et draps catalans ${ }^{1}$. C'est aussi une «entreprise» hasardeuse, pleine de risques politiques et coûteuse en hommes: confiée à un cadet de la Maison d'Aragon, elle peut très bien échapper au contrôle de la monarchie barcelonaise et la Couronne ne s'engage qu'avec prudence, alors que des volontaires affluent de Gascogne, de Castille, de Bretagne et même du lointain Hainaut ${ }^{2}$. L'expédition de 1392 a donc été préparée, avec les faibles moyens dont disposait l'infant Martin, duc de Montblanc, sur

1 Sur l'évolution politique et la conquête, F. Giunta, Aragonesi e Catalani nel Mediterraneo. I. Dal Regno al Viceregno in Sicilia, Palerme, 1953; V. D'AlessandRo, Politica e società nella Sicilia aragonese, Palerme, 1963, et maintenant P. CORRAO, Governare un regno. Potere, societa e istituzioni in Sicilie fra Trecento e Quattrocento, Naples, 1991. Sur le dessein de Martin, je citerai particulièrement la circulaire envoyée à Barcelone, Valence et Saragosse le 25 avril 1390: Nos, vayents lo gran be, bonor e profit que's pot seguir a la Corona d'A rag6.. e enquara a tots los regmes e terres del dit senyor (Jean Ier) e espocialment a aquexe ciutat per baver e obtenir lo dit regne (de Sicile)...; ACA Canc. 2091, f. 45.

${ }^{2}$ Je les cite dans H. Bresc, Les Gascons en Sicile, 1392-1460, communication au XIV Congresso di Storia della Corona d'Aragona La Corona d'Aragona in Italia nei secoli XIIIXVIII, Sassari-Alghero, 19-24 mai 1990, sous presse. 
tous les plans: militaire, par le recrutement de fidèles catalans et aragonais; diplomatique, par les ambassades qui ont semé la division entre les «seigneurs de Sicile»; économique, par la mise en gage des domaines du duc; mais aussi dans le domaine du symbolique: c'est la nébuleuse des ordres de chevalerie et de l'emblématique qui a été appelée à renforcer la solidarité autour d'un prince sans royaume, et à établir, avant même le départ en guerre, une fidélité que le rapport féodal ou la souveraineté ne pouvaient assurer.

Tant que la conquête ne serait pas accomplie, que le pouvoir de Martin ne serait pas implanté et fortifié par le couronnement de Martin le Jeune et de Marie, et les membres de l'expédition casés en Sicile sur les terres confisquées, l'expédition restait en effet un royaume errant, un État imaginaire, une cour et une administration sans base territoriale; elle aurait pu vivre son ambition comme une compagnie d'aventure, ennoblie par la conscience d'une cause dynastique juste, mais un ordre de chevalerie garantissait un propos gratuit et libre d'attaches contingentes. Cet ordre se définissait comme une "emprise», il unissait chevaliers, l'exploit militaire, la joute individuelle, l'esprit de Croissade ${ }^{3}$. Empresa, il était aussi amprisa, "entreprise", le mot même qu'Alphonse le Magnanime a choisi, à partir de 1421, pour qualifier la grande affaire de sa conquête de Naples.

Le texte des statuts de l'Empresa de la Correge sont conservés aux archives de la Couronne d'Aragon sous une forme qu'il faudra d'abord élucider; cette copie, qui semble unique, et les corrections qui lui ont été apportées permettent de définir la société chevaleresque, mise en place par l'infant Martin sous la forme d'une devise, comme un ordre, destiné à durer, et non comme une simple confrérie. Il s'agit, reprenant la classification de D'Arcy Boulton ${ }^{4}$, d'un ordre curial, et quasi immédiatament d'un ordre royal: les corrections apportées au texte transforment en effet l'institution du duc de Montblanc en la centrant sur Martin le Jeune, roi de Sicile. Il en résulte un ordre étrange, qui redouble les prescriptions pour les ajuster à cette figure bicéphale, où s'exprime toute l'ambiguité des relations entre le duc de Montblanc et son fils: le prince n'a jamais renoncé à ses propres droits sur la Sicile, mais c'est son fils et la reine Marie qui partagent le titre et la souveraineté. L'Empresa a enfin les apparences d'un ordre votif puisqu'elle place l'institution sous la promesse très forte, mais sans réalité, de la défense des dames.

${ }^{3}$ Cf. M. Pasteourenu, Traite d'béraldique, Paris, 1979, p. 218; badge ou «devise», comme marque propre, mais il faut prendre «emprise» en un sens plus large que l'emblème composé et allégorique des XVIe et XVIIle siècles.

${ }^{4}$ D'A. J. BoulToN, The Knights of the Crown: The Monarchical Orders of Knighthood in Later Medieval Europe, 1325-1530, Woodbridge, 1987. 
On la comparera donc aux ordres princiers de l'époque et de l'aire géographique qui touchent à l'Aragon et à la Sicile des dernières décennies du XIVe siècle, et plus particulièrement à ceux dont les statuts ont été conservés: la Banda d'Alphonse X de Castille (1330)', la Jarretière (1348), l'Étoile de Jean II (1351), le Neud de Louis de Tarente (1352) $)^{6}$, l'ordre de Sant Jordi de Pierre IV le Cérémonieux (1353) ${ }^{7}$; l'Écu d'or de Louis de Bourbon $(1367)^{8}$, la Nef de Charles II de Duras $(1381)^{9}$. Mais aussi aux devises et aux confréries, égalitaires comme Sainte-Catherine de Dauphiné (vers $1333)^{10}$, le Tiercelet poitevin (avant 1385) ${ }^{11}$, la Pomme d'or des seigneurs bourbonnais $(1395)^{12}$, ou plus hiérarchiques, l'Ecu vert à la dame blanche de Boucicaut (1399) ${ }^{13}$, le Dragon du comte de Foix (vers 1410) ${ }^{14}$, le Fer du prisonnier de Jean de Bourbon (1415) ${ }^{15}$. Ce choix n'est pas arbitraire: les contacts sont étroits entre les milieux chevaleresques et curiaux anglais, castillans et aragonais; le modèle français a dominé très largement la Catalogne de Jean Ier le Chasseur (qui a appelé son fils «Dauphin») et les chevaliers des ordres bourbonnais (souvent engagés dans d'autres compagnies, comme le Collier de Savoie) circulent largement, comme Boucicaut, Louis de Bourbon lui-même, Châteaumorand, Jean de Vienne, porteurs de modèles et de défis.

5 G. DAumet, L'ordre castillan de l'Écharpe (Banda), Bulletin hispanique, 25, 1923, p. 5-32. Et L. Tadeo Villanueva, Memoria sobre la orden de la Caballería de la Banda de Castilla, Boletin de la R. Academia de la Historia, 72, 1918, p. 436-465 et 552-574.

6 Comte Horace de VIel-Castel, Statuts de l'Ordre du Saint-Esprit au Droit Désir ou du Noeud, Paris, 1853.

7 R. SAInz de la MAzA, La Orden de San Jorge de Alfama. Aproximación a su bistoria, Barcelone, 1990, p. 167-180; statuts publiés p. 258-260.

8 A.-M. Chuzaud, La Cbronique du bon duc Loys de Bourbon, Paris, 1876, p. 8-15.

9 S. Pivano, Lineamenti storici e giuridici delle cavalleria medievale, Memorie della R. Accademia delle Scienze di Torino, ser. 2, 55, 1905, p. 255-336; Édition des statuts de la Nef p. 301-336.

10 U. Chevalier, Choix de documents bistoriques inédits sur le Daupbine, LyonMontbéliard, 1874, p. 35-39.

${ }^{11}$ M. G.A. VALE, A Fourteenth-Century Order of Chivalry: the Tiercelet, English Historical Review, 82, 1967, p. 332-341.

12 A. Bossunt, Un ordre de chevalerie auvergnat: L'Ordre de la Pomme d'or, Bulletin historique et scientifique de l'Auvergne, 64, 1944, p. 83-98.

${ }^{13}$ Le livre des faicts du bon Messire Jean Le Maingre, dit Boucicaut, éd. Michaud et POUJOULAT, 1836, p. 255-257.

14 P.S. LEW IS, Une devise de chevalerie inconnue crebe par la comte de Foix? Le Dragon, Annales du Midi, 76, 1964, p. 77-84.

is L. DOUËT-D'ARCQ, Choix de pièces inédites relatives an règne de Charkes VI, I, Paris, 1863, p. $370-374$. 


\section{LES STATUTS DE L'EMPRESA: TEXTE ET CONTEXTE}

La copie des statuts qui est ici publiée a été préparée sur parchemin dans les domaines ibériques de la Couronne d'Aragon, dans un pays de langue catalane, royaume de Valence, ou plus probablement Catalogne, puisque le choix du sanctuaire de l'ordre porte sur Montserrat ( $\S$ IV). La date de cette première rédaction, sans doute simple projet, se laisse aisément cerner: le duc de Montblanc prépare l'expédition de Sicile ( $\S \mathrm{X})$, et Martin le Jeune n'est pas encore roi titulaire de Sicile par son mariage avec Marie, célébré en 1390; le roi dont la souveraineté est invoquée au §III est donc le roi d'Aragon, Jean le Chasseur. Les statuts n'ont cependant pas été promulgués sous la forme, pourtant solennelle, de cette rédaction: ni souscription, ni scellement. Corrigés, selon les principes mêmes énoncés au §III, par la réunion plénière de l'ordre (ils étaient donc au moins six à accompagner le duc en Sicile), les chapitres ont été réutilisés comme minute, pour la rédaction, probablement, d'un autre acte qui n'a pas été retrouvé; sur cette minute, on a ajouté par la suite la notice de l'attribution de l'empresa à Huc de Santa Pau, le ler janvier 1396, comme si la charte originelle était devenue un embryon de chronique de l'ordre.

Le texte, mis au point donc entre 1385 et 1390 , a été modifié lors du chapitre statutaire tenu à Palerme le jour de la Saint-Jean 1392, probablement le premier dans l'histoire de l'Ordre, puisqu'il apporte les premières corrections aux statuts. La conquête, menée avec fermeté et brutalité, avait alors remporté ses plus grands succès. Toute l'île semblait soumise, l'armée avait débarqué le 22 mars à Favignana; la capitularion de Palerme, le 17 mai, avait suivi un court siège; l'arrestation par surprise, le lendemain, et l'exécution presque immédiate d'Andrea Chiaramonte, le ler juin, avaient scellé la collaboration des grands, ralliés au nouveau régime, le comte Guglielmo Peralta, chancelier de Sicile, et son fils Nicola, promu marquis de Mazara en 1392, le maître justicier Guglielmo Raimondo Moncada, comte d'Augusta. Tout allait en réalité basculer très vite et les conquérants, à la fin du mois de juin, se préparaient déjà à faire face à une insurrection généralisée: Guglielmo Peralta et le maréchal Galceran de Villanova partaient alors pour Girgenti en rébellion.

Les additions du chapitre de Palerme aux statuts de l'Ordre se manifestent par d'abord de petites corrections, qui prennent acte des modifications du contexte politique, et d'abord, §II, §VI, §XIII, du dédoublement de la compagnie: une fraction demeure en Aragon, tandis que l'autre accompagne le duc de Montblanc en Sicile; non sans naïveté, les rédacteurs enregistrent aussi, $\S \mathrm{X}$, le succès de l'expédition de Sicile et accordent une décoration, un plató, à chacun des membres de l'Ordre qui a participé à la conquête. Ils n'imaginent évidemment pas la longueur et la férocité de la 
guerre, les six années de guérilla, les pertes considérables (Ramon de Bages prisonnier, Galceran de Villanova tué), la rébellion et la confiscation des biens d'au moins deux d'entre les témoins du chapitre de 1392, Guglielmo Raimondo Moncada et Enrico Rosso. Ils prennent acte enfin de la promotion maintenant effective de Martin le Jeune: l'adjonction du § XIV impose un changement de numérotation des paragraphes suivants, et de menues corrections ( $\S \mathrm{XVI}$ et XVII, cette dernière enlevant toute cohérence à la phrase) qui ne changent en rien l'esprit des statuts. Enfin, les rédacteurs, le duc et ses compagnons, comblent une évidente omission: au §VII, ils ordonnent le port de l'empresa au jour de la Saint-Jean.

L'attribution de l'empresa à Huc de Santa-Pau, le ler janvier 1396, intervient dans un contexte de victoires, mais aussi de lassitude, presque d'épuisement, pour les conquérants comme pour les partis siciliens qui luttent ou qui négocient l'établissement d'un régime équilibré: Catane est le siège de la cour du duc de Montblanc depuis son entrée victorieuse du 9 août 1394; la révolte a été repoussée dans les zones périphériques, et la soumission de Palerme est en vue. Huc de Santa-Pau a été l'un des artisans de ces victoires.

Tel qu'il a été corrigé et complété, le texte des statuts de l'Empresa de la Correge présente, sous une forme brève, en un petit nombre de chapitres $^{16}$, un ensemble d'obligations et d'engagements classiques, qu'on peut regrouper en six parties: la défense des droits des dames et demoiselles, le cérémonial, le port de la devise, la composition de l'ordre, le secours mutuel en cas de rançons, l'usurpation de l'insigne. Ces 19 paragraphes ou chapitres précédés d'une introduction et suivis de deux paragraphes erratiques, qui se rattachent au quatrième groupe; l'invocation cependant, est originale: elle met l'ordre sous la protection de la Deesis, faisant intervenir comme patron de la compagnie non point un saint de fer, comme Georges (déjà protecteur de la milice instaurée par Pierre IV d'Aragon en 1353), Jacques le Tueur de Mores ou Michel, mais saint Jean le Baptiste, insistant donc fugitivement sur le devoir religieux. Cette insistance ne va pas sans contradiction avec la nature militaire des obligations des membres, ou avec le caractère purement laïque de la devise, sans aucune figuration religieuse, mais sans la touche mythologique qui donne à la Pomme d'or ou même au Noeud, fondé dans le château virgilien de Naples, un petit goût d'ésotérisme; le calendrier même de ses réunions, tel qu'il est révélé par la notice qui concerne Huc de Santa-Pau, ne

${ }^{16}$ Ce qui le rapproche, pour la même époque, des ordres princiers et des «emprises» féodales (11 chapitres pour l'Écu vert, 12 pour le Tiercelet, 13 por le Dragon de Foix, 14 pour le $F$ er du prisonnier) alors que les statuts de la Nef comprennent 154 articles; mais, à l'époque précédente, ceux de la Banda n'en comptaient que 22 et celui de Sant Jordi, 10 seulement. 
se plie pas aux nécessités religieuses: le ler janvier est la plus laïque des fêtes du cycle des Douze Jours.

Les deux premiers paragraphes expédient, rapidement et sans y insister trop, le thème de la défense des veuves et des jeunes filles en danger qui sert de prétexte à l'Empresa; les paragraphes III à VII établissent les règles, au demeurant classiques, du cérémonial: fête et chapitre général à la SaintJean, présence obligatoire des membres de l'ordre (à la requête cependant du duc, ce qui laisse entendre que le duc, trop occupé aux affaires, pourra ne pas convoquer le chapitre), choix d'une église prestigieuse, le monastère de Montserrat, et non fondation d'une collégiale ou d'une chapelle propre (comme Saint-Georges de Windsor, la Noble Maison de Saint-Ouen pour l'Étoile, Sainte-Catherine «à La Costa Saint-André» pour l'ordre dauphinois, etc.) pour l'acte rituel de la figuration des armes et des correges peintes, services religieux enfin pour les trépassés, à la charge, essentiellement, du duc. L'atmosphère, dans ces chapitres, n'est évidemment pas celle d'une confrérie, comme le Tiercelet: pas de service collectif, ni chapelain ni chapitre collégial, aucun devoir de piété particulier, aucun programme de vie, comme dans la Banda ou la Nef. Pas d'égalité, non plus, mais l'autorité du prince et le prestige de l'empresa dont il voudra, avec l'accord de ses compagnons, récompenser les gens de mérite. Le choix de l'Annonciade des Carmes de Trapani pour les services d'action de grâces (§ VI) manifeste la vénération des Catalans envers un sanctuaire mendiant et une statue miraculeuse particulièrement chers aux hommes de mer, et aussi, sans doute, une référence au point où, à l'ouest de l'île, s'est accompli le premier acte de la conquête, soumission de la ville, ralliement des Peralta.

Cette empresa est décrite avec précision au paragraphe VIII, et les conditions de son enrichissement de plaques émaillées et d'un dardalló sont énumérées aux paragraphes IX, X et XI. La minutie avec laquelle sont détaillées les expéditions et les batailles qui méritent les platons est comparable, quoique plus sommaire, à celle qui préside aux statuts du Noend, de la Nef ou du Tiercelet ${ }^{17}$ : il s'agit de transformer la correge en une véritable décoration qui manifeste au premier coup d'oeil l'excellence de celui qui la porte, d'en accumuler des preuves homogènes, sans cependant que Martin de Montblanc ait imaginé une insigne unique en forme de plaque capable

17 L'origine de la décoration complexe semble être la Nef, hiérarchisée (l'ancre pour une prise de ville, des cordes, des voiles pour une bataille, un timon pour une bataille de plus de 1500 hommes, des bannières pour achever); le Tiercelet est plus simple (et mal éclairci par l'editeur: qu'est-ce qu'un duve ou dime?): une serre d'or pour le voyage de Prusse, la bataille, le siège ou le simple gage; le Dragon, avec un diamant pour la joute, un rubis pour le corps a corps, une émeraude pour la bataille, un saphir pour la Croisade ou le voyage au Saint-Sépulcre, la turquoise pour le siège, est à la fois le plus rigoureux et les plus mondain, il s'inspire peut-être de la Correge. 
de porter, comme la nef ou le tiercelet, une gamme de garnitures significatives. C'est une simple courroie de cuir, qui recevra de petites plaques, passée autour du cou, «à demi découverte», dissimulée donc en partie par un vêtement sur lequel aucune précision n'est donnée. Cette troisième partie s'achève, après le paragraphe XII, sur le retrait pour "faute honteuse», par les paragraphes XIII et XIV, qui fixent à treize, puis, exceptionnellement, à quatorze, compris le roi de Sicile et le duc de Montblanc, le nombre des membres de l'ordre. Il s'agit donc bien d'un ordre, suivant le critère adopté par Olivier de la Marche: un nombre fixe de compagnons, des réunions à date fixe, des chapitres qui fixent les devoirs de chacun ${ }^{18}$. Ce n'est pas un corps d'élite: un drapeau existe bien, mais il s'agit seulement d'un pennon individuel, non d'une bannière collective et rien n'indique une «bataille» autour du prince ${ }^{19}$.

Les paragraphes XV, XVI et XVII fixent les termes de la solidarité réciproque entre le duc, puis le roi de Sicile, et leurs compagnons dans le cas, fort probable, où les combats entraîneraient la captivité de l'un d'eux. Martin de Montblanc est ainsi assuré de trouver des garants qui accepteront de se porter comme otages; lui-même, rejoint ensuite par le jeune roi, se met à la tête d'une mutuelle destinée à payer les rançons des compagnons prisonniers, jusqu'à une somme totale de 4400 florins par capture, 1000 par prince, 200 par compagnon. La solidarité, exclusivement financière de la part des princes, s'étend, pour les compagnons, à la prison volontaire, comme otages réciproques, en cas de nécessité. On peut comparer à ces engagements ceux que contiennent les statuts de la Nef, qui prévoient génériquement aide et soutien réciproque, mais surtout que la pourvéance du prince est de droit si un compagnon déclare sa pauvreté. Si le prince ne l'aide pas, il est délié de ses obligations militaires ( $\$ 61$ et 119: aide en toutes maladiies, prisons et pouretés; $§ 45$ : aux non poissans et povres le prince est tenu de pourveoir).

Les paragraphes XVIII et XIX eux aussi classiques, règlent sommairement le système des joutes et des défis: ordre votif par son fondement, l'Empresa devient aussi une société de joutes. Comme dans l'Ordre castillan de la Banda, le port non autorisé de la devise oblige les membres à relever le défi du combat singulier. Rien n'est dit sur une issue de la bataille

18 M. KeEn, Cbivalry, lu dans la traduction castillane La Caballeria, Barcelone, 1986, p. 241, analyse la conception attribuée par Olivier de la Marche à Philippe le Beau: l'emblème, sans statuts ni réunions, se donne sans limitation de nombre à des chevaliers, des dames, des demoiselles, des écuyers. Une confrérie a des statuts et célebre des chapitres, sans limitation de nombre. Un ordre, enfin, réunit statuts, chapitres et nombre clos.

19 La bannière collective apparaît dans la Banda, l'Etoile, le Noeud, la Nef; Boulton conteste à juste titre qu'elle guide un groupe particulier de combattants. 
négative pour le ou les membres de l'ordre qui auraient accepté le pas d'armes: considérerait-on l'affaire comme déshonorante, selon le précepte du paragraphe XII? Le chevalier étranger serait-il admis de plein droit dans l'Empresa? A la place laissée libre par le vaincu? L'absence même de précision me semble indiquer le caractère rituel et purement théorique de ces prescriptions.

L'institution chevaleresque établie par Martin de Montblanch réunissait plusieurs caractères: sous les apparences de la devise, ceux d'un ordre à la fois curial et votif et d'une compagnie d'hommes d'armes. Mais lesquels? Les biographies des premiers destinataires de l'empresa nous éclairent sur le sens que Martin et ses proches compagnons entendaient donner en 1392 à l'institution: Guglielmo Raimondo Moncada et Guglielmo Peralta font partie des pierres angulaires de la domination catalane dans l'île. Leur ralliement, précoce et décisif chez le comte d'Augusta, négocié patiemment pour les Peralta, se présente comme un retour aux fidélités d'origine de leurs familles; il s'agit en réalité du fruit provisoire de tactiques complexes, mais ce ralliement était nécessaire à Martin de Montblanc: il divisait le front baronnial et le privait en particulier de la direction politique que pouvait assumer la famille Peralta, alliée par mariage à la monarchie aragonaise de Sicile et héritière de la vocation au commandement des marquis impériaux de Haute-Italie (par le sang, ce sont des Saluces qui ont relevé le nom des Peralta. Mais ces siciliens (Moncada demeurant un peu moins sicilianisé que Peralta), plus disponible aux aventures étrangères, en Grèce par exemple, ou à la guerre de course en Sardaigne) n'étaient guère habitués à la pratique de cette fidélité choisie et de cette compétition au nom des idéaux chevaleresques. Pendant la durée entière de la domination des vicaires sur l'île, moment où la recherche de ces compagnonnages choisis aurait paru utile, une seule marque d'intérêt est perceptible à l'égard des ordres et des devises, et encore s'agit-il d'une démarche mondaine, qui cache mal l'autodéfense: en 1390, quand le duc de Bourbon revient de son expédition manquée contre Mahdia à la tête d'une armée multinationale d'aventuriers financés par Gênes, il saccage les ports de Sardaigne par vengeance; puis la fortune de mer rabat ses galères sur Messine et sur Trapani: Manfredi Chiaramonte fait bon visage aux soi-disants croisés; il les accueille pendant huit jours à Messine, les festoie et les défraie; à leur départ, il demande au duc de Bourbon de l'armer chevalier, ce qui montre bien la désaffection pour l'adoubement. Et le duc lui donne «une ceincture d'or de sa devise d'Espérance». Manfred les a ravitaillés et a offert aux chefs, le duc, le sire de Coucy, le dauphin d'Auvergne, le comte d'Eu, cinq beaux coursiers: il a manifesté dans cette occasion de remarquables qualités politiques, en écartant de la Sicile cette armée plus que dangereuse. Lui-même, atteint de podagre, n'est pas un combattant, mais il sait jouer, payer, plier pour ne 
risquer d'être proie ${ }^{20}$. Moncada et Peralta étaient eux-mêmes sans doute trop siciliens pour participer sans inquiétude à cette mise en place d'un état-major de proie.

Ils sont en effet entourés des chefs de guerre de l'expédition, Guerau de Queralt et Ramon de Bages, bientôt maréchal; Bernat Cabrera, capitaine général, amiral et comte de Modica, qui a "signé» les chapitres alors que le comte d'Aidone Enrico Rosso n'apparaît que comme témoin. Peut-être encore Pedro Sanchez de Calatayud, autre chef de guerre, Joan de Olzinelles, et le scribe royal Diego García. Ce ne sont pas, de toute façon, de simples compagnons, mais des capitaines et des secrétaires destinés à participer, la conquête faite, au régime même, à gouverner le royaume. Aucun doute n'est ici de mise, donc: l'ordre qui se cache sous les apparences de la devise a pour but d'attacher solidement une partie de l'état-major et les plus prestigieux des barons ralliés au duc de Montblanc, qui s'efface ostensiblement devant la jeune majesté de son fils. Le fondement en est politique. N'est-il que cela? Et surtout pourquoi avoir choisi cette forme complexe, multiple, incertaine, pour assurer des fidélités que nous savons changeantes chez les uns, soumises à leurs ambitions démesurées (les siciliens, Cabrera aussi), et fondées chez les autres sur la solidarité de l'entreprise commune de conquête, sur un noble butin?

\section{LE JEU, L'HONNEUR, LA GRATUITÉ}

Le perspective ludique n'est évidemment pas absente de la rédaction des statuts: la défense des veuves et des demoiselles fait partie de ces «conventions arbitraires et impératives», qualifiées par Bossuat de "puérilités", exaltées au contraire par d'autres comme «jeu primitif et sacré» de la guerre, elle-même fête cruelle ${ }^{21}$. Je ne prétendrai pas résoudre l'antinomie à partir de ce seul exemple, mais il est clair que le rituel est ici réduit à son minimum, que la convention est sans mystère, sans gravité, sans émotion. Seul, l'enjeu du combat singulier, dans le cas très improbable qu'un chevalier étranger usurperait la devise, présente le sérieux habituel, le danger de mort. Mais, dans une armée en guerre, un royaume d'Aragon engagé depuis plus de 50 ans dans la guerre féroce de Sardaigne, cet enjeu était assez banal. Aucun rituel vestimentaire, aucune gaieté soulignée par un rituel du banquet commun (implicite, sans doute, dans la réunion du 24 juin, mais d'autres statuts insistent sur la salle, le repas collectif), aucun

20 Chazaud, la Cósronique cit., p. 253-254.

${ }^{21}$ Par exemple M. Stanesco, Jeux d'errance du chevalier médieval. Aspects ludiques de la fonction guerritre dans la litterature du Moyen Age flamboyant, Leyde, 1988. 
effet de roman: à la différence de Louis de Tarente, de Charles de Duras, les statuts ne prévoient de faire raconter aucun exploit, ni d'en conserver la mémoire par l'écriture d'un "Roman» ou d'un «Livre des faits». On pourrait attribuer au réalisme légendaire des Catalans, au seny, l'absence de ces précisions. Ce serait négliger ce que tant d'autres documents contemporains confirment: en pleine conquête de la Sicile, les duels dans le camp de Martin, entre Guillem Chuan et Pere Ramon del Falgar, deux gascons, ou entre Darnius et En Saranyó ${ }^{22}$, la participation de Darnius aux entreprises de Boucicaut et l'obsession du fait d'armes notée par M. de Riquer ${ }^{23}$.

Sur un seul point, la prudence et le bon sens doivent cependant être relevés: le prince commande, il ne combat pas inutilement, ne participe pas aux expéditions lointaines, ne court pas de risque injustifié. On est très loin des précisions des statuts de la Nef sur la présence physique du souverain au cœur de la mêlée. Martin est donc autorisé à arborer les plaques émaillées sans avoir pris personnellement part aux batailles, trois à l'annonce de la convocation de l'armée pour le "passage de Sicile», un encore au départ de l'armada, les six derniers et le dardalló au débarquement dans l'île $(\S \mathrm{X})$.

Lattribution des plaques émaillées mérite une attention particulière: elle rappelle les décors complexes du Noeud et de la Nef, mais elle ne présente pas la hiérarchie complexe et minutieuse du second, ni la perspective eschatologique, centrée sur Jérusalem, qui les anime tous les deux. Dans

22 ASP Canc. 23, f. 107vo; 6.10.1394: Darnius. Ja sabets, coms vos nos supplicas que.ns plagues esser jutge de certes armes empreses de fer entre vos e En Saranyb e nos vos atorgam e.us assignam jomeda e apres lo dit Saranyb fon malalt per qu.os nos alongam la jomede en la bora que seria guarit e dispost per fer les dites armes, ara que lo dessús dit Saranyo s'es presentat a nos, prest de ésser a nostre jubi et baja sabut que vos entenets a partir breument d'aquest regne nos ba supplicat e request que sobre afo dejam provebir, per que nos ab la present vos manam que dins VIII jorns aprés presentacio de la present a vos fabedora siats e comparegats denant a nos per complir les dites armes... et ASP Canc. 37, f. 86; 2.7.1399: A l'amat e feel nostre En Guillem Chuan, salut e dilecti6, com per l'amat e feel nostre En Pere Ramon de Falgar senyor de Menerqua siats estat request de certes armes,... VIII dies apres que syam en la dita ciutat vos assignarem jomeda certa.

${ }^{23}$ Un gage de bataille entre le baron d'Isnello, le gascon Arnaud de Sainte-Colombe, dit «Colomat», «la petite colombe», et le sénéchal de Hainaut Jean de Werchin, est l'occasion d'une correspondance qui dure deux ans; Martin l'Humain accepte en 1405 d'en être le juge et d'organiser le camp. Si Jean de Werchin le prefere, Martin le Jeune poura le faire en Sicile; D. Girona Llagostera, «Itinerari del Rey En Martí (1396-1402)», Anuari de l'Institut d'Estudis Catalans, V, 1913-1914, p. 577. La bataille a lieu finalement le 30 mai 1407 à Valence; Werchin, Tanneguy du Châlet et leurs deux compagnons affrontent les deux Sainte-Colombe, Colomat et Peyrolet, Pere Montcada et Bernabò dell'Uovo. Le cérémonial est grandiose: Colomat est armé chevalier par la roi avant la bataille. Mais Martin est (aussi) avare du sang de ses chevaliers et il arrête le combat très vite; M. de RIQUER, Caballeros andantes españoles, Madrid 1967, p. 101. 
notre Empresa, le voyage de Jérusalem n'est qu'un pélerinage, au demeurant sans danger, et non la reconquête de la Terre sainte, royaume temporel des Angevins et gage d'un basculement millénariste. Il prend donc place entre les simples pèlerinages, Santiago, Sinaï, et les expéditions armées, à la fois Croisade, chasse sportive et imitation des romans arthuriens, de Prusse et de Livonie. Quant aux «batailles» et aux faits d'armes, la casuistique ici est simple: quatre figures seulement (bataille sans défi ni accord, bataille accordée à pied ou à cheval, combat singulier, combat à groupes égaux) et trois issues possibles pour les deux dernières figures (victoire, suprématie sans victoire, égalité). Et les récompenses sont d'une extrême monotonie; on est bien loins des complexités du Noeud et de la Nef, ou des décors chatoyants du Tiercelet et du Dragon de Foix. Ni or, ni pierres précieuses, deux simples plaques, l'une verte et l'autre rouge. Aucune recherche de symbolisme, comme aucun décor vestimentaire. Le vert et le vermeil ont pu être choisis pour distinguer l'Empresa des ordres à dominante bleue et or (la Jarretière, la Banda dans son évolution), mais le vermeil est lui-même d'une grande banalité (la Banda, l'Étoile, Sant Jordi, la Nef, la Bobine de Marguerite de Duras ${ }^{24}$ ), de même que la distinction or/argent pour signaler chevaliers et écuyers. Seul le vert présente une forte originalité: il annonce l'Écu vert de Boucicaut. Dans l'ensemble, donc, la recherche de règles contraignantes et de la distinction par le décor, dans cet ordre, reste rudimentaire, le cérémonial embryonnaire.

La perspective ludique, si elle n'est pas totalement absente, se réduit ainsi à un décor, un trompe-l'œil. L'honneur, au contraire, est exalté avec des techniques simples, presque universelles: pour chacun des compagnons, chevaliers ou écuyers, les armes peintes dans le sanctuaire choisi par l'ordre, la devise posée sur le drap funèbre et enterrée avec lui, la devise brodée sur un pennon, d'or pour le chevalier, d'argent pour l'écuyer, qui accompagnera sa bannière ou son pennon et qui sera posé sur sa tombe ( $\$ \mathrm{~V}$ et VII); on retrouve là l'écu et la bannière suspendus dans la chapelle de la Nef, les armes peintes dans la chapelle de Grâce Nostre-Dame du Fer du prisonnier; mêmes techniques aussi de «deshonnoirement»: la devise est retirée, mais en bloc; rien n'est prévu, comme dans la Nef ou dans le Dragon, pour un retrait gradué des plaques de décoration ou pour une restitution graduelle. Il n'est pas prévu non plus de châtiment infligé sur les armes du coupable,

24 T.F. RuIZ, Festivités, couleurs et symboles du pouvoir en Castille: les célébrations de mai 1428, Annales ESC, 1991, p. 521-546, insiste sur la combinaison du blanc et du vermeil dans les cérémoniaux royaux et les fêtes chevaleresques ibériques; ce n'est pas faux, mais pourquoi se placer dans la perspective intemporelle et initiatique de $M$. Eliade, rouge adamique et blanc féminin? Peut-être faudrait-il consommer l'enivrante symbolique avec quelque précaution. 
effacées ou retournées, mais il est implicite. La "faute honteuse» (§ XII) souligne la vulnérabilité de l'honneur individuel et collectif. Le déshonneur de chacun rejaillit en effet sur l'ordre et sur tous ses membres, mais la honte physique (défaite en champ clos), hautement improbable dans les conditions de 1392, s'efface ici devant la honte politique: la reddition d'un château ou d'une ville, la trahison envers le duc. Ou devant le non-respect des engagements: au $\S$ XVII, le refus de libérer le compagnon qui se sera porté comme otage et de payer sa rançon entraîne automatiquement la perte de l'empresa. La morale n'est donc pas celle du simple chevalier, de sa subjectivité irritée et précaire; une référence polie, permanente, et qui n'est pas innocente, est toujours faite au combat, à l'engagement physique, mais la référence ultime, dominante, est celle de la fidélité politique, dans un ordre régi par conseil, comme un bon gouvernement: les statuts prévoient, aux paragraphes I et II, la nomination de juges et d'experts, et la réunion du conseil pour examiner les cas des veuves et des demoiselles qui feraient appel au duc; aux paragraphes XI, XII et XIII, la présence du conseil lors de l'attribution des plaques honorables, lors du retrait et de l'attribution de la devise; au paragraphe XX, enfin, on prévoit un serment de loyal conseil prêté au prince sur ces affaires qui pourraient paraître mineures, mais où l'honneur est engagé.

En est-ce assez pour conclure que, loin d'être une simple décoration, un hochet, l'Empresa est une sphère de gratuité, gérée par ses propres lois, close sur elle-même, sur son choix arbitraire, qui fait de ses membres des êtres distingués par leur soumission volontaire, un lieu de perfection? Ce serait négliger le va-et-vient permanent entre l'Empresa et l'expédition de Sicile, le double rôle de ce conseil, à la fois juridiction d'honneur liée à la gestion interne de l'ordre et tribunal d'arbitrage entre les Douze compagnons et les princes sur le fait des rançons. La réalité la plus triviale, la prison, l'humiliation politique, la solidarité nécessaire pour chacun et pour le groupe, est intimement liée, tressée au formalisme de la représentation. Tous les membres de l'état-major du "passage de bonne aventure» de Catalogne en Sicile ne sont sans doute pas membres de l'ordre, mais les plus distingués, en effet, par leur fidélité personnelle au duc de Montblanc et par leur engagement personnel, militaire et financier dans l'entreprise. Un noyau plus actif, plus responsable, sur qui le prince peut également compter dans les moments les plus difficiles. Mais ce sont des hommes de proie, avides de butin, prompts aux dissensions. L'honneur et les apparences, vécues ou non, du jeu et de la gratuité ont ici le sens d'un ciment plus nécessaire et non moins efficace que la Jarretière, ou que l'Écu d'or pour la reconquête des terres de Bourbon; non pas un vaste rassemblement de fidèles, comme l'Étoile ou l'ordre de SaintGeorges de 1353, mais comme la Banda à l'origine, la Nef ou le Collier de Savoie, un groupe étroit, sûr, militant. 


\section{DE LA PRINCIPAUTÉ EN MARCHE AU HOCHET MONDAIN}

Tribunal militaire et juridiction d'arbitrage d'une entreprise de conquête, les termes pourraient être quelque peu ironiques, et renvoyer à l'image de la compagnie d'aventure d'abord écartée; mais une limite doit immédiatement être apportée à la comparaison. Une compagnie peut recevoir un vêtement qui l'apparente à une institution chevaleresque, ordre ou simple devise, mais sous la forme d'une confrérie. Une structure égalitaire ou faiblement hiérarchisée, des principes de fraternité et de défense mutuelle, éventuellement une durée limitée dans le temps. Le cas du Tiercelet, étudié par M. Vale, illustre le principe horizontal et la solidarité militaire: établi par Renaud de Vivonne et 17 autres chevaliers poitevins et saintongeais entre 1377 et 1385 , cet ordre se confond presque avec la force militaire de Louis de Bourbon et comprend des chefs de routiers. Même loyauté égalitaire, même élection du chef d'ordre en 1395 dans la Pomme d'or auvergnate, qui institue un système d'arbitrage interne destiné d'ailleurs à la faillite. La devise établie par Boucicaut en 1399, l'Écu vert à la dame blanche, aurait plus facilement pu glisser à la solidarité et à l'entreprise d'aventure: prévue pour cinq ans, sans base territoriale, elle se donne seulement un but ludique, celui de fournir constamment des combattants aux joutes et aux combats à outrance, mais elle a sans doute scellé la collaboration militaire des compagnons avec le maréchal. Plusieurs points de ses statuts offrent des ressemblances frappantes avec l'Empresa: le nombre, peu fréquent, des membres, treize, l'objectif affiché, la défense des dames et des demoiselles, la solidarité financière en cas de prison. Boucicaut a pu connaître les statuts de l'Empresa par ses compagnons gascons, mais il a pu aussi puiser dans un fonds commun encore mal connu.

Vertical et hiérarchique dès son origine, bicéphale après la reconnaissance de Martin le Jeune comme roi de Sicile et donc destiné à durer audelà de la simple vie de son fondateur, la devise du duc de Montblanc n'a pas été instituée sur la base territoriale qui donne une cohérence aux confréries et aux ordres qui se déploient au sein d'une principauté ou sur une vaste région frontière, comme la Pomme d'or et le Tiercelet. Martin, largement possessionné en Aragon, à Valence et en Catalogne, ne disposait pas d'un apanage continu: il ne pouvait pas en appeler ni à la défense de la "patrie», ni même à la lutte contre les Mores, comme le fera en 1403 un autre infant, Ferdinand, le futur el de Antequera, dans son ordre de la Jarre et de l'Etole. Sa principauté est un camp, un droit, une ambition, l'image en réduction de ce que sera l'empire d'Alphonse le Magnanime. Comme Martin, Alphonse saura utiliser pour sa conquête de l'Italie les potentialités de l'institution chevaleresque. Il fera ainsi broder un étendard semblable à ceux des compagnies d'aventure aux couleurs et à la devise de l'ordre fondé 
par son père: ce sera donc un étendard rouge aux lys d'or qui guidera l'armée du Magnanime vers le triomphe napolitain ${ }^{25}$. La similitude des deux entreprises éclaire rétrospectivement l'efficace présumée de la devise comme ciment de l'armée de conquête. On s'étonne même que Martin n'ait pas utilisé son emblème sur les étendards, et l'ait cantonné aux pennons des chevaliers. Il est vrai que le choix du duc de Montblanc était, de ce point de vue, malheureux: une simple courroie de cuir n'était guère remarquable; ni franchement scandaleuse, comme la Jarretière, ni chargée d'un sens religieux comme la Jarre aux lys de la Salutation angélique, la correge faisait partie de l'arsenal innombrable des liens, fers de prisonnier, nœuds, colliers, bandeaux, écharpes, qui marquent la dépendance honorable. Sa représentation manquait sans doute de beauté graphique, et sa lecture aurait été difficile. On ne s'étonnera donc pas de voir Martin l'Humain, roi d'Aragon, lui préférer d'autres devises, tout en la conservant pour en décorer quelques fidèles, et surtout des dames valenciennes.

Ordre curial, l'Empresa de la Correge coïncide, sans doute imparfaitement, avec le conseil du prince: en 1392-1396, il nous est difficile de préciser, faute d'une liste exhaustive des membres du conseil et de connaître avec certitude les Douze de l'Empresa, la plage commune aux deux institutions $^{26}$. Notons seulement que le jeune Huc Santa-Pau est conseiller dès 1391, comme d'ailleurs son père, que Moncada, maître justicier, est attesté comme membre du conseil en 1396, Ramon de Bages, alors maréchal, en 1397. Bernat Cabrera, amiral et maître justicier après la félonie de Moncada, l'année suivante; il ne fait aucun doute que Guglielmo Peralta, alors chancelier titulaire, en ait fait partie en 1392. Cette coïncidence partielle est sans doute un facteur de concurrence, de compétition et de dynamisme. L'ordre ennoblit l'organe essentiel autour duquel se forge l'Etat. Non point l'Etat sicilien, qui a ses propres institutions assez fortes pour avoir tenu pendant l'kanarchie baronniale» (mais aussi parce que les barons les ont utilisées et sans doute, jusqu'à un certain point, vénérées), mais l'Etat de conquête "catalan». En ce sens, il faut comparer l'Empresa et la Nef: les statuts de Charles de Duras sont beaucoup plus précis sur les

25 A. Giménez Soler, Itinerario del rey Don Alfonso de Aragón y de Nápoles, Valence, 1909, p. 135; Gaète, 17.2.1436, le roi demande un standart vermell ... de la manera e forma que los capitans de gent d'armes los acostumen de portar en Italia, en lo mig del qual baja una terrafa ab los lirs tal com nos la acostuman de portar en nostra empresa e divisa et tot l'estandart sia sembrat de flors de lirs solament ab los parons los quals terrafa e flors sien totes d'or. Sur l'ordre de la Jarre de la Salutation angélique, devenue l'Étole et la Jarre en 1412, simple devise sans rien de militaire, v. A. MCKAY, Don Fernando de Antequera y la Virgen Santa Maria, Homenaje al professor Juan Torres Fontes, Murcia, 1987, II, p. 949-956.

${ }^{26} \mathrm{P}$. CorraO, Governare un regno cit., publie les listes de conseillers connus, aux. p. 463-464. 
devoirs de fidélité des membres de l'ordre, sur le respect de la majesté du prince; ils intériorisent profondément la pratique spirituelle (jeûnes, offices, vêtement noir du vendredi) pour la mettre au service du prince: l'ordre fait fonction de tribunal, les chevaliers y ont un devoir de délation des crimes commis par les autres membres contre la fidélité ( $\$ 98)$, une véritable confession des fautes y est instituée ( $\$ 97$ et 100) et le secret enveloppe la vie interne de l'ordre.

C'est l'institution chevaleresque mise au service de l'État lourd de tradition normande et souabe, dans une position assurée: Charles tient déjà Naples et le royaume. L'Empresa n'a pas les moyens, ni sans doute encore les ambitions, de tout régir et de tout plier; le dynamisme de Martin de Montblanc est dans sa souplesse: unir les ambitions en un faisceau efficace, sinon cohérent, pour acquérir. La conquête ne laisse pas le temps de surveiller les fidélités et, de fait, l'insurrection serpente en même temps, mais l'acquisition constante, les ralliements, les distributions de biens, font de l'Etat de conquête une boule de neige qui finit par étouffer et écraser la rebellion.

La conquête réalisée, et avant même que tous les barons siciliens soient soumis, la mort de Jean Ier, sans héritier depuis celle du Dauphin, appelait Martin de Montblanc au trône d'Aragon; le prince quittait alors sa création sicilienne. Non sans une lenteur qui marque son attachement à son œuvre personnelle et sa confiance dans l'habileté de Marie de Luna pour écarter les périls de l'entreprise du comte de Foix ${ }^{27}$. Roi d'Aragon, couronné à Saragosse après une préparation méthodique, particulièrement sur le plan du symbolique, qu'allait-il faire de la devise du duc de Montblanc? Il semble que Martin n'ait pas senti, dans la forêt de l'emblématique flamboyante, l'intérêt de maintenir fermement en un corps unique et prestigieux les fidélités de ses proches. Sans doute n'en avait-il alors nul besoin. L'Empresa ne sera donc pas pour l'Aragon ce qu'elle était en Sicile, une autre Jarretière, éprouvée, comme l'ordre d'Angleterre, par la guerre de conquête, la guerre honorable et profitable. La pratique de Martin conduit au déclin, puis à la disparition de la Correge. A. de Fluvià atteste que la devise a encore été distribuée en 1397, en 1400 et en 1408 à des barons catalans, respectivement Ferrer d'Abella, Jaume de Prades et Pere de Torrelles ${ }^{28}$. Une recherche -évidemment incomplète- dans le fonds immense des registres de chancellerie de Martin a permis de retrouver plusieurs lettres

27 Il ne quitte la Sicile que le 10 janvier 1397 pour gagner Cagliari, Alghero, Ajaccio, Avignon, et ne retourne en Catalogne, après cinq années d'absence, que le 18 avril; Girona, Itinerari, Anuari cit., IV, 1912-1913, p. 81 sq.

${ }^{28}$ Article Corretja de la Gran Enciclopedia Catalana, qui ne prend en compte l'ordre qu'au retour de Martin en Catalogne. 
d'attribution de l'empresa à des dames de l'aristocratie, complétant ainsi les informations données par A. Javierre Mur: Violant de Montagut, épouse de Mossen Ramon Boyl ${ }^{29}$, Violant de Montsoriu et Beatriu de Montagut ${ }^{30}$, Beatriu, épouse de Mossen Bernat Scorna ${ }^{31}$, Ysabel, épouse d'En Pere Fabra $^{32}$, Violant, épouse de Micer Ramon Soler et leur fille Alienor ${ }^{33}$, Violant, fille d'En Ramonet Castellar et Agnès, fille d'En Guillem de Blanes ${ }^{34}$, Maria Lladró de Villanova ${ }^{35}$, Joana Pujada, épouse de Joan Escrivà, et Guisabel Pujada ${ }^{36}$, Maria de Mur ${ }^{37}$, et encore Isabel et Leonor de Pertusa, Violant de Heredia ${ }^{38}$. Ces familles sont, en majorite, valenciennes; elles ne sont pas de premier plan dans l'aristocratie du royaume (les Mur, cependant, sont alliés aux Cervelló, aux Carròs, aux Pinós, les Lladrò sont vicomtes de Xelva) mais elles occupent une place honorable dans les offices royaux (les Blanes, les Pujada). Certaines, qui sont d'ailleurs alliées, Castellar et Balnes, ou ennemies (Soler), jouent un rôle brillant dans les luttes civiles, les bandositats de Valence. Les Fabra participeront au Paso Honroso de Suero de Quiñones... C'est dire la soif de dignité et d'aventure chevaleresque de cette moyenne aristocratie urbaine. Le ressort de l'attribution de la devise et, sans doute, de la demande du récipiendaire relève cependant maintenant de la vanité la plus universelle: le port de la correge fait en effet échapper son titulaire aux ordonnances somptuaires, en particulier municipales, édictées contre le luxe. Chaque lettre spécifie clairement le droit de porter pierres précieuses, perles fines, draps d'or et de soie, brocards, velours, ou, plus simplement, tout vêtement et ornement, à condition qu'il accompagne l'empresa ${ }^{39}$. La féminisation évidente de la Correge lui fait rejoindre d'autres «emprises», l'Ermine des ducs de Bretagne (vers

29 A. JAvierre MUR, Maria de Luna, reina de Aragon, Madrid, 1941, p. 202.

30 Arxiu de la Corona d'Arag6, Barcelone, Canc. 2167, f. 179 et 179 vo; Saragosse, 15.6.1398

${ }^{31}$ Ibid., f. $179 v^{\circ}$; Saragosse, 30.8.1398.

32 Ibid., f. 179v'; 12.9.1398.

33 Ibid., f. $179 v^{\circ}$; 4.10 .1398$.

34 Ibid., f. $179 v^{\circ} ; 21.11 .1398$.

35 A. JAVIERre MUR, Maria de Luna, cit., p. 209; 8.1.1399.

36 ACA Canc. 2170, f. 138 $\mathbf{v}^{\circ}$; mai 1399; information que je dois à l'amitié de Maria Teresa Ferrer, que je remercie aussi por les reférences qui apparaissent aux notes 42 et 44 , et pour avoir bien voulu reviser le texte catalan des Statuts, et enfin pour ses remarques et ses suggestions.

37 ACA Canc. 2168, f. 137 ${ }^{\circ} ; 22.5 .1402$, de Valence (lettre non expédiee).

38 A. JAvierre Mur, Maria de Luna, cit., p. 115.

39 Lettre à Maria Lladrò de Vilanova: axi que vos, portant aquella (empresa) sobre vostra persona, puixats portar pedres, perles fines, draps d'aur e de seda, brocats d'aur e de velluts et tots altres ormaments e arreus que.us volrets, e.us serà vist faedor. Qualsevol ordinations, estatuts, fets e faedors en contrari no contrastants. 
1382), le Porc-Epic d'Orléans (1394), la Jarre des Transtamare fondée en 1403 comme devise masculine et féminine, le Dragon de Foix; ces "pseudoordres", selon l'expression de Boulton, expriment un besoin social intense, qui ne doit pas être sans rapport avec la popularité des "Neuf Preuses». Compensant le déclin de sa fonction militaire, quelques fantasmes guerriers ne hantent-ils pas le crépuscule de la Correge?

Ce déclin de la Correge a conduit Martin à créer de nouvelles devises ou à en réactiver d'anciennes: en 1399, Guillem et Joan Pujada, frères de Guisabel et fils du batlle general de Valence, reçoivent du roi celle de la Garrotera, à porter sur la jambe gauche, au-dessus du pied ${ }^{40}$. S'agit-il d'une imitation de l'ordre anglais? Ou plus simplement d'un emblème votif? La Jarretière présente en effet tous les caractères d'un défi courtois, donc ambigu: élément du vêtement féminin, il affiche un lien secret, dont il défend la pureté d'intention par les armes, faisant taire par la force les médisants. Plus tard, Martin a distribué la devise royale de la Croix; deux lettres, à Francesquina de Cardona et au comte de Cardona, l'attribuant au fils de la première, attestent qu'elle ne pouvait être «imposée» que par un chevalier et $a$ nobles bomens $e$ cavallers ${ }^{41}$; une autre ordonne cependant à un damoiseau, Juan Fernández de Vilafranca, de la remettre à Pere de Thous, maître des arsenaux de Séville ${ }^{42}$. Cette incohérence signale sans doute que l'intérêt de Martin l'Humain pour ses devises s'est dispersé. Cette Croix était sans doute le vieil ordre de Saint-Georges institué par Pierre le Cérémonieux pour la défense de son royaume et tombé graduellement lui aussi au rang de devise honorable: il est vrai que si Saint-Georges visait à constituer une milice d'élite toưfours mobilisable, l'ordre avait été distribué, dès 1353, à de grands seigneurs étrangers, comme les Mérode. Vers 1404, le roi d'Aragon a encore créé une devise, dite de la Leona Parda, la léoparde, adressée à Martin le Jeune, en septembre 1404, puis à En Bernat Joan de Valence l'année suivante ${ }^{43}$. Encore une devise honorable, mais non un ordre chevaleresque, et une autre preuve de l'absence d'une politique du symbolique cohérente et ferme après le retour en Catalogne.

$$
\text { *** } *
$$

40 ACA Canc. 2170, f. 138v'; 15.5.1399.

41 Girona Llagostra, Itinerari, Anuari cit., V, 1913-1914, p. 539; 28.11.1403; com cavaller deja fer la primera imposicio en cascú de la dita creu.

42 ACA Canc. 2181, f. 114; 12.6.1406. L'hypothèse de Boulton, The Knights of the Crown cit., p. 284, semble donc justifiée: l'ordre de Sant Jordi (que Boulton postdate de vingt ans) s'est bien maintenu jusque sous Martin l'Humain.

${ }^{43}$ M. de RiQueR, Heraldica Catalana des de l'any 1150 al 1550, I, Barcelone, 1983, p. 219. 
L'Empresa du duc de Montblanc met en lumière l'extraordinaire ductilité du symbolique, sa capacité à exprimer en même temps les vanités mondaines, la verdeur et la force de l'ambition la plus terre à terre, l'idéal d'une société isolée, particulière et irréductible, et sa disponibilité à changer dans le temps de système de référence, à épouser tour à tour les contenus d'un ordre curial, mais simplement ducal, puis d'un ordre royal, enfin d'une simple devise, d'un emblème vite dégradé et abandonné. Ces qualités ont fait de la Correge un outil remarquable d'unité et d'efficacité. Elles n'ont pas interdit les trahisons, ni les luttes de factions qui ont entraîné reculs politiques, retours en Catalogne et sécessions des grands seigneurs de l'île. Pour eux, l'apprentissage de ces fidélités choisies restait à faire; plus froidement réalistes, ils ne cachaient pas leurs ambitions, ne rêvaient pas d'imiter Tables rondes et royaumes imaginaires. En réalité, c'était leur royaume, la Trinacrie du Xrve siècle, qui apparaissait depuis 1260 à l'Europe en expansion des noblesses ambitieuses, française, gasconne, savoyarde, catalane, castillane, portugaise bientôt (l'expédition de Ceuta menacera d'abord la Sicile) comme un royaume imaginaire exposé en proie, une conquête glorieuse parée des prestiges de la littérature et du folklore, et, d'une certaine manière, ennoblie, comme l'Athènes de Pierre le Cérémonieux, par une archéologie elle-même imaginaire.

Les Vicaires n'avaient pas découvert cet instrument, ou trop tard; sans doute leur manquait-il aussi le projet ambitieux, l'esprit d'aventure qui s'accommode mal avec une attitude nécessairement défensive. Leur position ressemble à celle de la France des Valois qui n'élabore qu'avec difficulté un grand ordre royal, alors que ses ducs multiplient ordres et emblèmes, face à une Angleterre agressive dont l'ordre royal réunit les capitaines de la guerre glorieuse et profitable. Psychologiquement, ces gens rassis que sont les nobles siciliens, plus juges et gouvernants qu'ambitieux et militaires, ne devaient guère se sentir à l'aise au milieu de ces catalans aventurés et à l'horizon illimité.

La Correge contribue donc, depuis cet angle modeste, mais significatif, de la géographie et de la politique imaginaires du monde médiéval, à éclairer quelques-uns des problèmes posés par le pullulement des ordres. On peut saisir ces derniers comme des projets fugitifs d'accroissements durables, comme des mouvements, créateurs d'Etats, de pouvoirs, d'avenir sur le modèle de l'imaginaire, plutôt que comme des instruments de défense de l'acquis. Une affaire de jeunes, en quelque sorte, comme les «abbayes» dont sortent les armées suisses à la même époque. Un expansionnisme sans limites et sans crainte, au service de la centralité monarchique, qui le pilote, le manœuvre et en tire gloire et profit. 


\section{TEXTE DES STATUTS}

\section{ACA Pergaminos Juan II apéndice}

En nom de Nostre Senyor Déu e de la Verge Madona Sancta Maria, mare sua, e del beneyt Sant Johan Babtista. Lo Senyor infant En Martí, duch de Montblanch, ab alcuns nobles,/ cavallers e scuders, per fer servey a Déu e a la sua mare beneyta e al dit sant, han feta la Empresa de la Correge en deffensió e manteniment dels drets de les dones vídues e pubils, per ço que, si prejudici / d'açí, avant los ere fet, que.s puxen recórrer al dit senyor qui aquells e aquelles ab lo voler de Déu ensemps ab los de la dita empresa entenen a deffendre e mantenir en lurs drets en la / forma e manera contenguda et expressada en los següents capítols.

I Primerament, que, com la dita empresa sia feta per deffensió de dones vídues e pubils e lo dit senyor duch e tots los altres de la dita sua empresa los hajen a deffendre en lur dret, si.s seguia que.l dit senyor / duch e qualsevol dels altres faessen tort en sa terra a dona vidua o pubil se accorie al dit senyor que li faés justícia, que tantost lo dit senyor la haje a fer o assignar / hi jutge no sospitós a coneguda de dos de la dita empresa, qui semblantment no fossen sospitosos al dit senyor ne a la dita dona ne pobil. E semblantment hajen a fer tots los altres a / conexença del dit senyor duch e de dos no sospitosos segons dit és. E que sobre aço acompir e servar, en cas que.ls dessús dits ne fossen negligents, lo dit senyor los ne pogués / forçar e destrènyer.

II Item, que.I dit senyor duch e tots los altres de la dita empresa hajen a deffendre dones vídues e pubils en lur dret, però que aquell se haje a regonèxer per aquell savi que.l dit senyor hi assignarà. E, feta / la regonexença per lo dit savi, sia solicitat e request per lo senyor duch aquell qui farà lo dit tort que dins certs dies ho torn a loch, e si no.u vol fer apres la dita requesta, sien appellats per lo dit / senyor duch tots aquells de la dita empres qui dins lo regne seran e, si no.y són tots, pus sien de VI ensús, ab aquells VI ensemps sia per lo dit senyor ordonada e acordada la manera / quina e qual se tendrá en fer la defensió demunt dita, peró que açó no sia entès en lo regne de Sicilia ne axí matex aquells qui seran en lo dit regne de Sicilia no sien tenguts fer la dita defensió / a dones vídues ni pubils del dit regne d'A rago ${ }^{44}$.

III Item, que, en honor e reverència del dit monsenyor sant Johan, lo dit duch face en lo seu dia festa cascun any, en què tots aquells de la dita empresa

44 De peró à Arago, rajouté d'une autre plume. 
hajen a ésser presents, si lo dit senyor los ne requerrà / per temps, ne si ells ne seran disposts de lurs persones, ne si són dins la senyoria del senyor rey, e lla ells puxen adobar e affegir en los dits capítols tots ço e quant los plaurà, pus que sien I de VII avant, no comprès en lo dit nombre lo dit senyor duch. E si per ventura entre any lo dit senyor hi volrà res mudar, tolre, corregir o anadir que pugue fer ab IX de la dita empresa e lo dit senyor lo X.

IV Item, que les armes del dit senyor duch e de tots los altres de la dita empresa se hajen a pintar ab les correges al monestir de madona Santa Maria de Muntserrat e los noms d'aquells, posant / los en orde segons que primerament seran en la dita empresa reebuts et admesos.

V Item, que per cascun de la dita empresa qui morrà, haje a fer dir lo dit senyor cent misses e tots los altres de la empresa vint e aquestes se hajen a fer dir tantost lla on sabran la mort del dessús dit, si loch / serà convinent, e fer un anniversari. E sobre lo drap que portarà e aprés en la tomba tenga estesa aquell qui mort serà una correja ab aytants platons com haurà lo dia de son obit.

VI Item, que.l senyor duch sobredit haje a fer dir cascun any en lo dit monestir de Montserrat, per ço que Deus lo vulla endreçar, vint misses e tots los altres e cascun d'ells VII. Es entés emperb / aquells qui seran en lo regne d'Arago e aquells qui seran en lo regne de Sicília a la esgleya de la Nunciada de Träpena ${ }^{45}$.

VII Item, que quant que quant alcú de la dita muyre, se haje a metre sobre sa tomba aprés la bandera o panó de ses armes I altre penó ab la dita correja, ço és cavaller d'or e scuder d'argent ab / tots platons com haurà lo dia de sa mort.

VIII Item, que la correja se haje a portar per lo coll a volta e mija descoberta e que la hajen a portar tres dies la setmana, ço és lo dimenge, lo dimars e lo dijous $e$ lo dia de sent Joban de juny, ${ }^{46}$ e en cas que allo no facen, hajen a dar a / menjar aquell dia a I pobre là on lo dit senyor no sia. En en lo loch on lo senyor duch sia, hajen a dar I florí a la sua almoyna.

IX Item, que en la correja no gossen portar plató alcú ne dardalló sinó per les coses seguents, ço és per lo viatge de / Peruça ${ }^{47}$ I plató vert, per lo viatge del Sant Sepulcre I plató vert, per lo viatge de Sant / Jacme I plató vert, per lo viatge de Santa Caterina I plató vert, e per lo viatge de Niflant ${ }^{48}$ I plató vert, e per batalla en terra o en mar I plató vermell, e per tota besunya arrestada que / meten peu a terra pus que sien de L ensús de cada part, puguen metre I plató vermell. E semblantment és entes de besunya de cavall pus emperò sia arrestada.

$\mathrm{X}$ Item, que si alcú de la dita empresa se combat cors a cors e vençrà, pugue metre tres platons vermells en la correja. E si cas és que la batalla no sia menada a fi, e lui serà juiciat lo millor, puga / metre dos platons vermells, e si sera juiciat egual, ço és que alcun no haje aventatge pus, però, armes s.i facen, puga metre I plató vermell. E si.s combaten tants per tants e vençran, / ne puguen metre dos vermells. E si la batalla no vendrà a fi e serà juciat haver lo mellor o egual, ne

4s De Es à Trapena, rajouté d'une autre plume.

${ }^{46}$ De $E$ lo à juny, rajouté d'une autre plume en interligne.

47 Lire Prufa, Prusse.

48 Liefland, la Livonie. 
puguen metre I vermell. E com al senyor duch no sia axí expedient com / als altres de fer los viatges ne altres coses demunt especificades per metre los platons, és ordonat, axí que, com a Déu plaura, que ell pos son estandart o taula per acordar les gents que ab ell deven anar en lo seu benuyrat passatge de Sicília, que lavors ne puxa metre tres, ço és dos vermells e un vert. Item al dia que.s reculliran, ne puxa metre un vermell. E com a Déu plaurà que sia arribat e haja presa terra en Sicília, que lavors los puxa metre tots e dardalló e que cascú per lo dit viatge, faent-lo ab lo dit senyor ne puxa metre I vert et feta la conquesta del dita regne I vermell ${ }^{49}$.

$\mathrm{XI}$ Item, que aquell que haurà complit metre $\mathrm{X}$ platons en la correja entre vermells e verts puga metre dardalló en la correja, però que alcú no gos metre plató en la correja sens lo senyor duch / qui, ab consell d'aquells cavallers que li parrà, axí de la empresa com altres, ho haja determenar.

XII Item, que si alcun de la empresa, ço que Déus no vulla, era vençut en camp clos o havia retut castell o vila, o havia feta tal falta envers lo senyor duch que la empresa ne fos envergonyada, a aquest aytal lo senyor duch / li puga e deja levar la dita empresa e d'aquí avant no la puga recobrar ne haver, però la falta aquesta haje ésser primerament juciada per lo dit senyor duch e VI de la dita empresa elegidors per lo / dit senyor.

XIII Item, que.I dit senyor duch sia cap e senyor de la dita empresa e aquella puxa donar a qui ell plaurà, però ab voluntat de III sens ell, e no la puxe donar sinó a XII, e ell lo XIII. E si alcú ne / mor, hi puxe metre altre lo dit senyor, però que haja apellar tots aquells de la dita empresa qui seran dins lo regne on ell sera $\grave{~}^{\text {so }}$ e si no.y vendran, que la puxe dar ab III de la dita empresa. E si lo dit senyor no era en lo dit regne d'Aragó, que ab aquells qui ab ell seran presents la puxe donar lo dit senyor.

XIIII Item, que.l senyor rey de Sicília, no contrestant lo dit nombre, sia de la dita empresa ab lo qual són ara XIIII, e per tal car no és entenció del dit senyor duch ne dels altres de la dita empresa que puxen ésser més avant del / dit nombre de XIII, sinó ara tant solament que s'és fet per reverència del dit senyor rey, volen et ordonen que, en cas que alcun ne moris o fallis de la dita empresa, que fossen tornats en lo dit nombre de XIII e no n.i posqués / ésser més alcun ne / aquell nombre posque / ésser crescut / altre vegadas!.

$X V{ }^{32}$ Item, que en cas que.l dit senyor duch fos pres, ço que Déus no vulla, que tots los de la dita empresa hajen a treballar per sa deliurança e metre.s en hostatges, si per lo dit senyor ne seran requests.

$X V I^{53}$ Item, que, en cas que alcú de la dita empresa sia pres, ço que Deus no vulla, ${ }^{34}$ haje a donar, fo es lo senyor duch $M$ florins de Florència e lo senyor rey altres ${ }^{55}$ mil florins per son rescat e tots los altres CC florins lo I a l'altre. E que aquells

49 De Et à vermell, rajouté d'une troisième main.

so On ell será en interligne.

"1. De XIIII à vegada, rajouté en interligne et dans la marge droite.

32 Corrigé sur XIIII.

33 Corrigé sur $X V$.

54 Rayé lo senyor ducb dessusdit.

ss De go es à altres en interligne. 
sien / tenguts a dar dins dos meses que.n seran requests per aquell qui serà pres o per altre qualsivol en nom seu. Es entès emperd, si aquell qui serà pres de la dita empresa paga per sa / finança tant o plus com munta la ajuda dessus dita, e, en cas que tant no munt, que pro rata sia abatut de la ajuda. E d'açò lo presoner haja a fer sagrament de dir la veritat.

XVII ${ }^{36}$ Item, que, si era cas que alcú de la dita empresa fos pres e havia mester hostages per sa deliurança, que aquell que ell demanaria de la dita empresa, exceptat los dit (sic) senyors, sia tengut anar tener / hostatges per aquell qui serà pres, per què aquell qui volria que per ell sia tengut lo dit hostatge haja a fer seguretat a l'altre, aytal com la demanerà, que possible sia, de la possibilitat de la qual haja a / conèxer lo dit senyor duch ab III de la dita empresa, e que, si lo presoner vendrà a menys a aquell qui per ell tendrà lo dit hostatge, que.l senyor duch ab los altres sia tengut traure / aquell qui y sera romàs per los hostatges, pagant cascú pro rata segons la forma de la ajuda faedora entre ells. E hagen a perseguir lo senyor duch e.ls altres aquell per qui's tendrà / l'ostatge cant, tan longament e tan fort, tro que sia cobrat e pagat lo dit hostatge ab tots dans e messions que per aquesta rahó seran fetes. E lo dit senyor li haja a levar e tolre / la ampresa e no la li puxe tornar, ans encontinent la haia a donar a altre.

XVIII ${ }^{57}$ Item, que, si alcú portava la dita empresa dintre la senyoria del rey d'Aragó e de Sicília ${ }^{58}$, sinó aquells a qui lo senyor duch la darà, que lo dit senyor ab los altres de la empresa haja a fer per tal manera que la / pos.

XVIIII ${ }^{59}$ Item, que, si algun altre la portava en la forma demunt specificada e requeria alcun o alcuns de la dita empresa de fer armes, que les hajen a complir ans que lexar la dita empresa.

$X X^{60}$ Item, que tots aquells qui hauran prestar consell en alcuna cosa que toch la dita empresa facen sagrament que bé e leyalment segons lur bona consciència hi prestaran lur consell, tota favor oy rancor / e mala volentat a part posada.

$X X I^{61}$ Item, que, si per ventura en los presents capitols o en alcun d'aquells apparran o exiran alcuns dubres o escuretats, que aquelles puxa enterpretar e declarar lo dit senyor duch ab VI de la dita empresa.

Fuerunt firmata capitula in Civitate Panormi die festi beati Johannis XXIIII junii anno $\mathrm{LXXXXII}^{\circ}$ per dominum ducem nomine suo et nomine domini regis jamdicti, Guillelmum Raymundum de Montechatheno ${ }^{62}$, comitem Guillelmum de Peralta ${ }^{63}$, Geraldum de Queralto ${ }^{64}$ et Raymundum de Bages ${ }^{65}$, etc. ${ }^{66}$.

36 Corrigé sur XVI.

\$7 Corrigé sur XVII.

s8 Et de Sicilia en interligne.

39 Corrigé sur XVIII.

60 Corrigé sur XIX.

${ }^{61}$ Corrigé sur $X X$.

62 Guglielmo Raimondo III Moncada, comte d'Augusta, auteur de l'enlèvement de la jeune reine Marie en 1379, chef corsaire en Méditerranée occidentale en 1388, maître justicier de 1392 à 1395, marquis de Malte en 1396, rebelle en 1397, mort en 1398.

63 Guglielmo Peralta, comte de Caltabellotta, vicaire réginal depuis 1378, chancelier en 1392, rebelle en 1393, mort en 1396 ou 1397. 
Testes firme predictorum comes Errigo Russi ${ }^{67}$ et Petri (sic) Sanxes de Calataiubio ${ }^{68}$. Testes firme Bernardus de Craparia ${ }^{69}$ qui firmavit dicta die, Johannes de Ulzinellis miles ${ }^{70}$ et Didacus Garcia de scribania domini ducis jamdicti.

Die sabbati prima januarii anno dominice Incamationis $\mathrm{M}^{\circ} \mathrm{CCC}^{\circ} \mathrm{XCV}^{\circ}$ in civitate Cathanie dominus dux concessit nobili Hugoni de Sancta Pace militi, consiliario et camerlengo suo ${ }^{71}$, emprisiam supradictam, quiquidem nobilis promisit complere in capitulis supradictis, obligans etc. juravit etc., homagium dicto, renuncians etc. Testes venerabilis abbas de Scarpio ${ }^{70}$ et Bernardus Berengarius de Perapartusa ${ }^{73}$.

${ }^{64}$ Guerau de Queralt, père de Pere, conseiller et chambellan; il avait servi le roi de Chypre en 1378. Mari de Beatriu Olzinelles, il est le parent de Joan. Il recevra le comté de Caccamo, enlevé aux Chiaramonte. Mort pendant l'expédition de Sicile.

${ }^{65}$ Ramon de Llupià alias Bages, chevalier roussillonnais, chambellan et maréchal; il a servi en 1484 contre les Compagnies en Roussillon; capturé en 1394 par Antonio Ventimiglia; il recevra Caltavuturo, puis Solanto en 1397, peu avant son retour en Catalogne (1398); il est alors capturé par des pirates; revenu en Sicile en 1402 au secours de Martin le Jeune, il se voit restituer Caltavuturo; il est alors "l'un des principaux de nos barons collatéraux»; il quitte définitivament la Sicile en 1405.

${ }^{66}$ Ce paragraphe et le suivant sont de la même main que les corrections.

67 Enrico Rosso, comte d'Aidone, puis de Sclafani; en 1405, il achètera Caltavuturo à Ramon de Bages.

68 Pere Sanchez de Calatayud, chevalier valencien, chef de guerre et diplomate, majordome de Martin de Montblanc; il recevra Gagliano et San Filippo d'Argirò en 1392, puis les restituera à la Cour.

69 Bernat Cabrera, comte d'Osona, capitaine général de l' «armada» de 1392; il reçoit le comté de Modica et la charge d'amiral enlevés aux Chiaramonte; en 1397, il organise et finance l'expédition de secours en Catalogne. Rebelle en 1403, mais pardonné, il est le principal adversaire de la reine Blanche de Navarre pendant l'interrègne.

70 Joan d'Olzinelles, chevalier catalan, parent de Bernat, trésorier de Pierre IV le Cérémonieux.

71 Huc IV de Santa Pau, généralement nommé Huguet pour le différencier de son père (mort en Sicile en 1393), conseiller de Martin, recevra Vizzini en 1397 et mourra en 1398; voir E. I. MINEO, Egemonia e radicamento della nobiltà militare catalana in Sicilia dopo il 1392: l'esempio dei Cruilles e dei Santapau, in Commercio, finanza, funzione pubblica. Stranieri in Sicilia e Sardegna nei secoli XIII-XIV, ed. M. Tangheroni, Naples, 1989, p. 89-128.

72 Frère Francesc Borradino, abbé du monastère cistercien de l'Escarp, au diocèse de Lérida, accompagne Martin en Sicile. Clémentiste, il joue un rôle important, comme juge et arbitre des affaires ecclésiastiques; en 1396, il est chargé de la levée de la décime triennale concédée par Benoît XIII. S. Fodale, Scisma ecclesiastico e potere regio in Sicilia I Il duca di Montblanc e l'episcopato tra Roma e Avignone (1392-1396), Palerme, 1979, à l'index.

73 Bernat Berenguer de Perapertusa, damoiseau roussillonnais de famille d'origine languedocienne (Pierrepertuse), hussier d'armes de Martin; il conduit una troupe en 1392 avec son frère Amill; châtelain du Sacré Palais de Palerme en 1393; il sera durement réprimé par le duc en février 1394 pour n'avoir pas bien gardé un château; il se fixe à Girgenti l'année suivante comme châtelain de la tour de la marine; en 1397, il reçoit en fief le château de Tavi et, en 1399, épouse la veuve de Nicola Bondelmonti, qui achète le château et le fief de Misilcassimo, puis, en 1404, la fille de Costanza Ventimiglia, Ylaria. Il a pris la nom de Castellar. 


\section{RESUMÉ}

L'emprese de la Correge instituke par Martin, duc de Montblanc, se présente comme une devise qui attache au duc un groupe fermé de chevaliers, dédié à l'exploit militaire, au combat singulier, au pelerinage, au passage armé, autour du voeu central, la défense des dames. Les statuts, adoptés avant 1390 et adaptés en 1392, après l'occupation de Palerme, manifestent un ordre curial et royal; en vingt-et-un paragraphes, sous un décor influencé par les modèles français, les statuts construisent la solide architecture d'un groupe de pouvoir et de combat, qui mène l'entrepise de la conquête de la Sicile, et garantissent solidarité, paiement mutuel des rançons, juridiction d'arbitrage, unité et efficacité au service de l'État de conquête centré sur Martin le Jeune et gouverné par le duc. $\lambda$ son accession au trône d'Aragon, Martin de Montblanc n'a pas maintenu la devise dans sa fonction politique; il l'a distribué à des barons catalans, mais aussi aux dames de l'aristocratie, créant par ailleurs ou réactivant d'autres hochets mondains, sans désormais véritable politique du symbolique, dont l'État aragonais n'avait pas besoin.

\section{SUMMARY}

The empresa of the Correge instituted by Martin, Duke of Montblanc, appears as a motto that attaches, to the Duke, a reduced group of knights, who dedicate their lifes to military exploits, single combats, pilgrimages, armed passage, around the central vow, defense of the ladies. The status, adopted before 1390 and adapted in 1392, after the occupation of Palermo, are the manifestation of a curial and royal order. Composed by twenty-one paragraphs and strongly influenced by French models, the status build up the solid anchitecture of a power and combat group, who seeks the conquest of Sicily, and guarantee solidarity, mutual payment of ransoms, arbitration jurisdiction, unity and efficiency at the service of the State of conquest whose soul is Martin the Young and that is governed by the Duke. When Martin of Montblanc ascended the throne of Aragon, he did not maintain the motto in his politic function. He attributed it to Catalan barons, but also to the ladies of the aristocracy, creating or reactivating on the other hand other pleasures of Society, without, from then on, cultivating a real politic of symbolism, that the Aragonese State did, in fact, not need. 